\title{
Research on Online Insulation Testing of Power Battery of New Energy Vehicles
}

\author{
Yuan Xu \\ College of Electrical \\ Engineering, Southwest Minzu \\ University, \\ Chengdu, China
}

\author{
Huazhang Wang \\ College of Electrical \\ Engineering, Southwest Minzu \\ University, \\ Chengdu, China
}

\author{
Jiacheng Li \\ College of Electrical \\ Engineering, Southwest Minzu \\ University, \\ Chengdu, China
}

\begin{abstract}
The insulation performance of new energy vehicles is an important factor in the normal operation of vehicles. This paper designs a voltage injection-type insulation detection based on the traditional detection.Based on the python language combined with the library provided by NI-visa, it can achieve high integration and meet the national GB/T 18384.1-2015 standard. Experimental results show that the insulation detection system can accurately test the insulation performance of new energy vehicles and meet the new energy vehicle offline detection standards.
\end{abstract}

Keywords: insulation test;new energy vehicles;power battery;insulation resistance;py-visa

\section{INTRODUCTION}

With the rapid development of the automobile manufacturing industry, domestic and foreign automobile manufacturers and major parts suppliers have shifted their business development focus to new energy vehicles, and the electrification of vehicles has become a development The inevitable trend [1].Whether it is a hybrid vehicle or a pure electric vehicle, the biggest feature is the use of high-voltage battery technology. The new energy vehicle power supply system is a typical AC/DC hybrid ungrounded system [2]. In order to meet high-power output, the output voltage of new energy vehicles is generally higher than $300 \mathrm{~V}$, and some even reach 720 V.In severe cases, it may cause safety accidents such as leakage and fire, which will seriously threaten the personal safety of drivers and passengers [3].Strengthening the accurate detection of the insulation performance of the power battery of new energy vehicles is a very important technology to ensure the safety of drivers and passengers in new energy vehicles.

Python has the characteristics of object-oriented, concise and efficient, high portability, and good scalability, and is widely used in computer information processing[4].Py-visa is a Python package toolkit for NI-VISA, which provides support for the test system to implement different interfaces of similar instruments, accesses hardware attributes in the interpreter under the Python script environment, and uses the relevant information of the VISA driver library [5].This paper designs a new energy vehicle power battery online insulation detection system based on Python language and $\mathrm{C}$ language.

\section{ON-LINE DETECTION METHOD OF INSULATION}

The vehicle chassis is the main body of the connection. The insulated electrical system of the new energy vehicle chassis is shown in Figure1. The insulation performance of the vehicle high-voltage system is reflected by detecting the insulation of the positive and negative bus bars of the power battery of the new energy vehicle [6].The power of the highvoltage system of the new energy vehicle is provided by the battery pack, and the motor and the motor controller, air conditioning, brake and steering assist constitute the load electricity in the system. This article mainly introduces the insulation performance test of the power battery part.
Common methods for testing the insulation performance of new energy vehicle power batteries include signal injection, balanced bridge, unbalanced bridge, and marginal insulation detection methods [7].

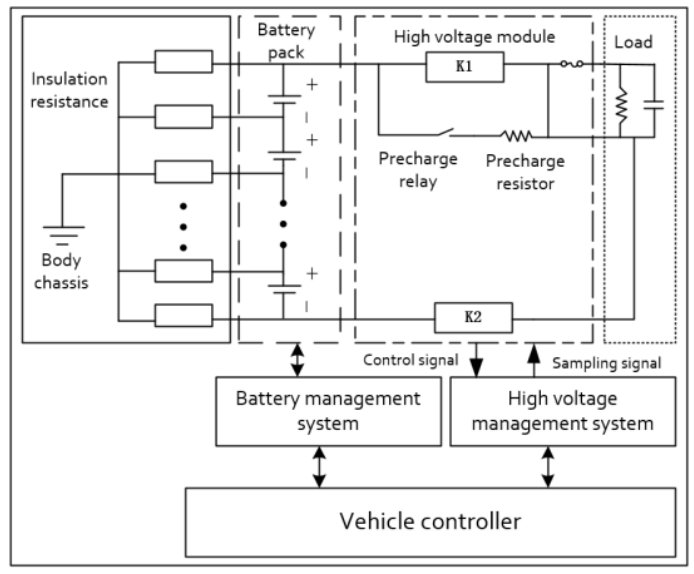

Figure. 1 Vehicle chassis insulation electrical system

The signal injection method of new energy vehicle power battery insulation detection is to transmit the signal to one end of the positive and negative charge and discharge interface of the power battery through a signal generator, load a Hall detection sensor ${ }^{[8]}$ on the load, and connect the other end to an insulation detection device. In the state of good insulation performance, the load input current is equal to the detection output current, and the direction is opposite, and the Hall element has no signal output. When an insulation fault occurs inside the power battery, part of the AC current forms a current loop with the ground through the ground resistance of the insulation detection instrument, and the currents on both sides are no longer equal, and the Hall sensor sends out a voltage signal alarm. The injection of low-frequency signals will increase the DC voltage ripple of the vehicle system and affect the power supply quality of the DC high-voltage system.

The balanced bridge method means that the resistance values of the upper and lower bridge arms are artificially incorporated, so the circuit changes introduced into the vehicle system are also balanced. Although the structure of the balanced bridge method is simple, the upper and lower bridges may still maintain a balanced state when the 
insulation resistance of the positive and negative bus change in the same proportion and grounded. At this time, errors are prone to occur when the insulation resistance is detected ${ }^{[9]}$.

The unbalanced bridge method is to add the unbalanced resistance of the photoelectric control switch to the insulation detection circuit to detect the insulation resistance of the positive and negative bus bars to the ground ${ }^{[10]}$. The limitation of the unbalanced bridge method is that it can only be used when the bus is charged. It can't work normally when the bus is not charged.

The active insulation detection method uses the PWM signal to control the isolation transformer, and injects high-voltage DC signals between the positive and negative buses of the battery and the car body ${ }^{[11]}$ to detect the insulation resistance. Although the insulation resistance can be detected when the positive and negative buses are not charged, however, the momentary high voltage during detection may have a great impact on the vehicle circuit itself.

\section{DETECTION PRINCIPLE AND SYSTEM PLAN:}

According to the GB/T 18384.1-2015 national standard, the insulation resistance of the power battery is usually defined as "if the power battery is short-circuited at a certain point between the stages, the resistance corresponding to the maximum leakage current". The standard states that the minimum insulation resistance of power batteries is $100 \Omega / \mathrm{V}$, and the safe value is $100-500 \Omega / \mathrm{V}$. The insulation resistance value is divided by the nominal voltage of the DC system of the vehicle, and the result should be greater than $100 \mathrm{~V}$ to meet the safety requirements. If the value is lower than this value, it is determined that the vehicle has an insulation failure ${ }^{[12]}$.

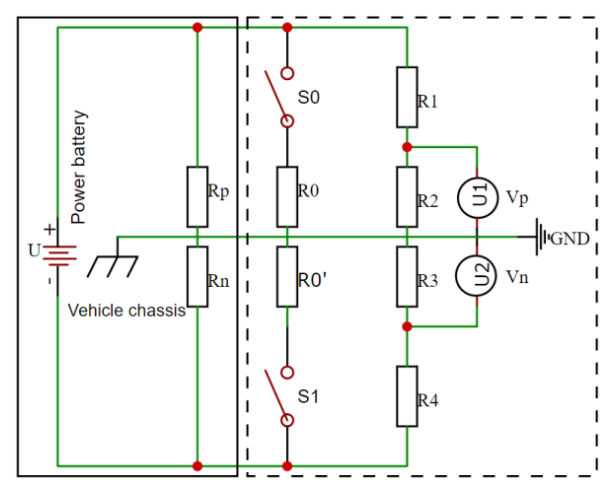

Fig. 2 Schematic diagram of equivalent circuit of vehicle chassis

The principle of the equivalent circuit for measuring the vehicle chassis is shown in Figure 2, where $U$ is the power battery voltage, $R_{n}$ and $R_{p}$ represent the insulation resistance of the positive and negative bus bars to the ground, respectively. The dashed block diagram is a pure electric vehicle insulation resistance monitoring circuit model. Inside the dashed block diagram is a new energy vehicle power supply model, where $\mathrm{R}_{0}, \mathrm{R}_{0}$ ' are standard bias resistors, and $\mathrm{R}_{0}$, $\mathrm{S}_{1}, \mathrm{~S}_{2}$ form a bias resistor network. $\mathrm{R}_{1}$ and $\mathrm{R}_{2}, \mathrm{R}_{3}$ and $\mathrm{R}_{4}$ constitute a measuring voltage divider circuit, $U_{p}$ is the positive voltage to ground, and $\mathrm{U}_{\mathrm{n}}$ is the negative voltage to ground. When measuring, first disconnect $S_{1}$ and $S_{2}$ to obtain the voltage values $U_{p}$ and $U_{n}$ of the positive and negative bus to the ground, and then determine whether R0 is in parallel with $R_{p}$ or $R_{n}$ according to the magnitude of $U_{p}$ and $U_{n}$.

If the measured value of $U_{p}$ is greater than or equal to the value of $U_{n}$, close $S_{1}$ and open switch $S_{2}$, and measure a set of positive and negative bus-to-ground voltage values $U_{p}$ and $U_{n}$ '. The calculation of the insulation resistance value $\mathrm{R}_{\mathrm{i}}$ of the DC high-voltage system can be obtained by the circuit principle The formula is as follows :

$$
\begin{gathered}
\frac{\mathrm{U}_{\mathrm{p}}}{\mathrm{R}_{\mathrm{p}}}=\frac{\mathrm{U}_{\mathrm{n}}}{\mathrm{R}_{\mathrm{n}}} \\
\frac{\mathrm{U}^{\prime} p}{R_{\mathrm{p}} / / R_{0}}=\frac{\mathrm{U}^{\prime}{ }_{n}}{R_{n}}
\end{gathered}
$$

Solving the simultaneous formulas (1) and (2),

$$
\mathrm{R}_{\mathrm{n}}=\left(\frac{\mathrm{U}_{p} \mathrm{U}^{\prime}{ }_{n}}{\mathrm{U}^{\prime}{ }_{p} \mathrm{U}_{n}}-1\right) R_{0}
$$

Since $U_{p} \geq U_{n}$, then $R_{p} \geq R_{n}$, and $R_{i}$ takes the smaller resistance value $R_{\mathrm{n}}$.

When the total voltage of the battery pack is too low or the battery pack has an open circuit fault, the high-voltage DC signal is used to inject additional high voltage between the positive and negative buses and the car body through the isolation transformer, so that the insulation detection circuit can also be used when the positive and negative buses are not charged. Calculate the insulation resistance. Figure 3 is a schematic diagram of voltage injection insulation detection. The circuit in the dashed frame is the sampling circuit of the insulation detection device, and the injection signal generation part. $\mathrm{R}$ is the sampling resistor, and the appropriate sampling signal is obtained by adjusting the resistance of the rheostat. After obtaining the final selected resistance value in the experiment, it will be applied to actual working conditions in the form of fixed value resistance. In the injection signal generating circuit part, the single-chip microcomputer outputs PWM signal to control the on-off of the IGBT, and induces a $700 \mathrm{~V}$ high-voltage signal on the secondary side of the transformer, and then obtains a DC high-voltage signal through RC filtering. The transformer uses a fly back converter with input and output isolation, and only one filter capacitor is needed for output filtering. At high voltage output, avoid high voltage inductors and high voltage freewheeling diodes. The power transistor is turned on at zero current and the turn-on loss is small. And the diode is turned off at zero current, and the reverse recovery problem can be ignored.

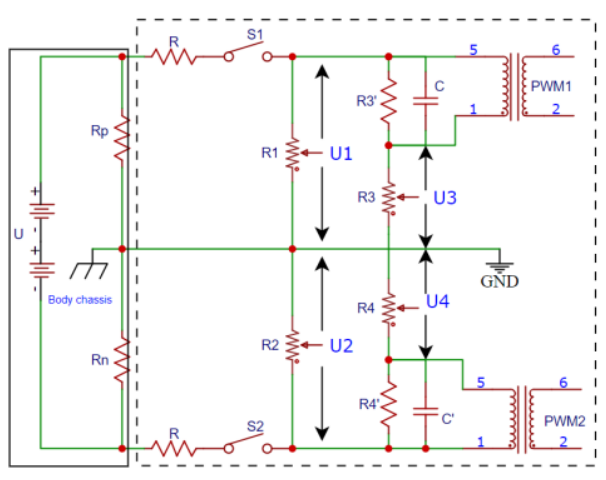

Fig. 3 Schematic diagram of voltage injection insulation detection

$S_{1}$ and $S_{2}$ are closed. Sampling the voltage of the resistors $R_{1}$ and $R_{2}$, after conversion, the voltages $U_{p}$ and $U_{n}$ on the ground insulation resistance of the positive and negative buses 
can be obtained. Then the total voltage $\mathrm{U}$ of the DC system is obtained.

$$
\mathrm{U}=U p+U n
$$

$S_{1}$ is closed and $S_{2}$ is open. Assuming that the voltage sampled at both ends of $R_{1}$ is $U_{1}$, combined with the current direction shown in the figure, the equation is obtained:

$$
U=\frac{U_{1}\left(R_{1}+R\right)}{R_{1}}+R_{n}\left[\frac{\mathrm{U}_{1}}{\mathrm{R}_{1}}+\frac{\mathrm{U}_{1}\left(\mathrm{R}_{1}+\mathrm{R}\right)}{\mathrm{R}_{1}+\mathrm{R}_{\mathrm{p}}}\right]
$$

$S_{1}$ is closed and $S_{2}$ is open. Assuming that the voltage sampled at both ends of $R_{2}$ is $U_{2}$, the equation is obtained by combining the current direction in the figure:

$$
\mathrm{U}=\frac{\mathrm{U}_{2}\left(\mathrm{R}_{2}+\mathrm{R}\right)}{\mathrm{R}_{2}}+\mathrm{R}_{\mathrm{p}}\left[\frac{U_{2}}{R_{2}}+\frac{U_{2}\left(R_{2}+R\right)}{R_{2} R_{n}}\right]
$$

Combining equations (4) and (5) to solve the equations can obtain the insulation resistance of positive and negative buses to ground. Analyze the working process of insulation resistance detection with positive and negative buses with and without electricity. When the positive and negative buses are electrified, the switches $\mathrm{S}_{4}$ and $\mathrm{S}_{5}$ are controlled by the singlechip microcomputer, so that $\mathrm{R}_{1}$ and $\mathrm{R}_{2}$ are connected in parallel with the insulation resistance $R_{p}$ and $R_{n}$ of the positive and negative buses to be measured respectively [13]. The external measuring resistance $R_{1}=R_{2}=R_{a}$. Taking the negative pole of the battery as the reference ground, first sample the total battery voltage to obtain $\mathrm{U}$. Then the $\mathrm{S}_{4}$ switch is in the off state, and the measuring resistor $R_{1}$ is connected in parallel with R. At this time, the single-chip microcomputer collects the voltage of node 1 and obtains $U_{p}$. Open switch $S 4$, close switch $\mathrm{S}_{5}$, connect the external resistance $\mathrm{R}_{2}$ and the resistance $R_{n}$ to be measured in parallel to obtain $U_{n}$.

\section{4 .HARDWARE DESIGN:}

The insulation resistance monitoring system of pure electric vehicles mainly completes the three major functions of measurement, communication and cutting off the high-voltage circuit. In order to meet the system functional design requirements, reduce the interference caused by hardware circuit errors, and improve the convenience and high integration of user operations, the system hardware structure is shown in Figure 4. The main components include the main control system, measurement control instruments, and program-controlled power supply, High-voltage wiring harness, etc.

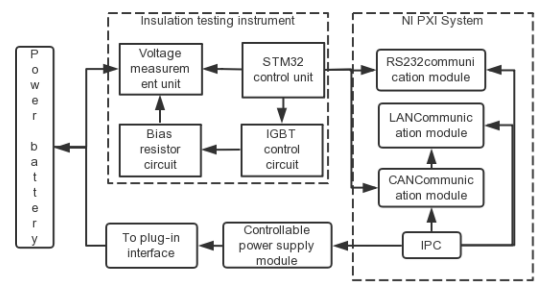

Fig. 4 hardware structure of insulation detection system

The main control system adopts the NI-based PXI expandable system to ensure the interoperability of instrument-level interface standards of different manufacturers' products. It can expand the control interfaces such as GPIB, LAN, RS232USB, etc. according to the difference of different instrument control interfaces ${ }^{[14]}$. In order to meet the characteristics of product diversification and various parameters, the hardware architecture of the debugging system adopts the "computer platform + control line" mode, which can realize interface standardization (including signal interface and hardware interface), test instruments can be interchanged, and test channels can be configured Wait.

The insulation detection instrument unit adopts the STM32 high-performance single-chip microcomputer introduced by ST, which itself has built-in 16-channel 12-bit high-speed ADC, which can simplify the hardware of the system [15]. The communication module reliably and efficiently realizes the internal and external communication between the detection system and the battery management system through the CAN bus. The main control chip drives a bridge unit composed of three elements $\mathrm{K} 0, \mathrm{~K} 1$, and $\mathrm{R}$ to achieve the measurement of the voltage between the positive and negative bus to the ground; the bridge unit uses an IGBT switch with a withstand voltage of $1000 \mathrm{~V}$, which has a fast response speed. The voltage of the measured resistance is input to the external ADC after the resistance divider, and then digitally isolated into the single-chip microcomputer. The insulation resistance value is calculated by the collected voltage and the known standard resistance value. When the positive and negative bus of the power battery are not charged, the PWM drive circuit works to drive the transformer to generate high voltage and complete the insulation resistance detection when the positive and negative bus are not charged. The high-voltage cut-off module calculates according to the measured positive and negative bus-to-ground voltages against electric vehicle safety standards, and controls the high-voltage contactor to cut off the high-voltage circuit in time to ensure the safety of the insulation detection of electric vehicles.

\section{SOFTWARE DESIGN AND TESTING:}

Python is an easy-to-use scripting language. Based on the above principles, scripts are written in Python language through GPIB, RS232, Ethernet, USB and other interfaces to communicate with various measuring instruments and test equipment [16]. The entire software system adopts a modular design to achieve The integrity of the insulation detection cycle, the calculation of the insulation resistance value, the fault alarm and other functions. According to the overall function of the system, the whole set of software consists of the following modules: main program module; CAN and RS232 communication module; data processing module;alarm program module; edit each parameter in order to be applicable to the protocol of different BMS manufacturers, such as The ID, byte position, scale factor and unit of the CAN message are edited and the XML file is finally generated [17]. The software flow chart is shown in Figure 5. Click the insulation safety detection module on the main interface to enter its subinterface. The user first clicks the open button on the left to start the insulation detection hardware module, and then clicks the start detection button to display the current DC voltage and positive in the reading display column. Resistance of negative pole to ground. The main function modules of the system software platform include personnel login, product selection, debugging module, and report output data query. After the system is powered on, the system completes initialization and generates a square wave signal to control the push-pull circuit. In order to improve the sampling accuracy, the CPU judges the size of the feedback signal in real time. If the feedback signal is too small, the lift circuit is enabled. After the collection is completed, the insulation resistance 
value is calculated, According to the calculated value, judge the insulation fault level alarm.

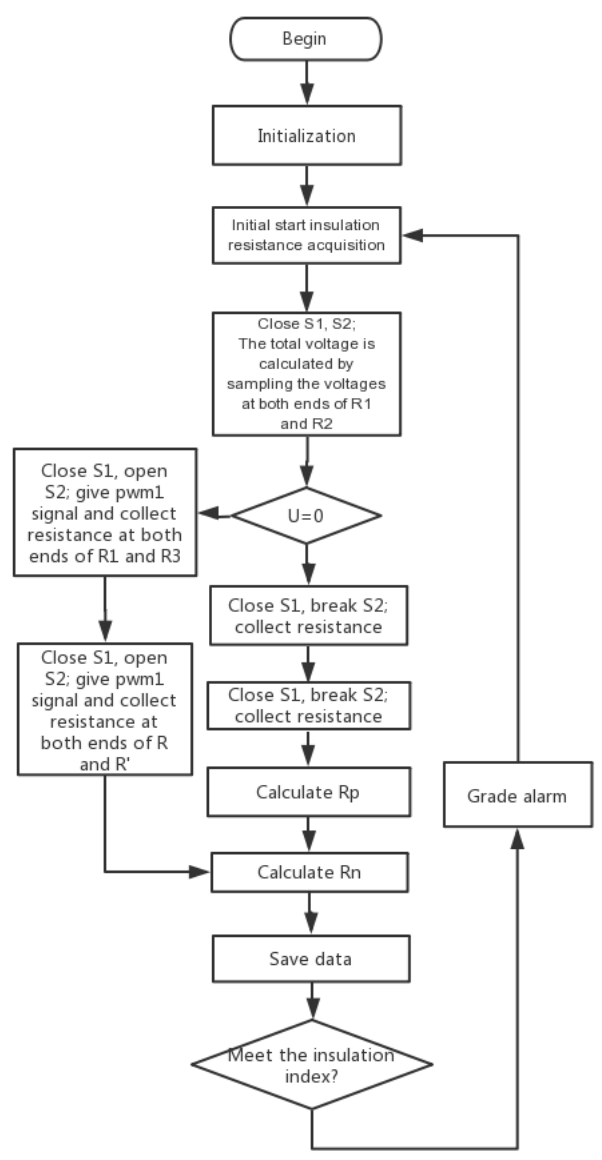

Fig.5 software flow chart

The insulation detection instrument is based on the $\mathrm{C}$ language programming of the STM32 single-chip microcomputer, carried out under the MDK integrated development environment, and mainly completes the $\mathrm{AD}$ measurement program. First, collect the total voltage $U$ of the battery pack, and judge whether the bus is charged by the collected $U$. If $U>0$, use the balanced bridge method to detect the insulation resistance. After the insulation resistance $\mathrm{Rp}$ and $\mathrm{Rn}$ are calculated, the smaller one is compared with the threshold value. When the insulation resistance value is less than the threshold value, the insulation fault location is performed; when $\mathrm{U}=$ At 0 o'clock, the PWM signal is used to drive the DC source to boost injection to detect the insulation resistance.

\section{CONCLUSION}

Aiming at the shortcomings of traditional passive insulation measurement methods for new energy vehicles, this paper proposes a voltage injection insulation testing method. This method fundamentally solves the shortcomings that the symmetrical insulation fault of the power battery to the ground cannot be measured. The insulation performance between the circuit and the vehicle chassis. In the process of off-line testing of new energy vehicles, it can accurately conduct insulation testing of power TVs, improve off-line standards, and enhance vehicle driving safety. The next step of research will focus on real-time detection, improve its detection accuracy and reduce the size of the equipment, so that it can be integrated into the battery management system for secondary verification and real-time grasp of vehicle operating conditions.

\section{7 .REFERENCES}

[1] Zhu Minhui. Connecting the future helps the spare parts supplier behind China's new energy Weilai es8 [J]. Automobile and accessories, 2018, 000 (002): 50-51

[2] Liu Junfeng, Wu Jialei, Zhu Xiangkai, et al. A hybrid high voltage DC and high frequency AC pure electric vehicle electrical system: 2018

[3] Yang Kun, Yang Lin, Shi Yixin, et al. Single point insulation fault location method for electric vehicle power battery pack [J]. Automotive engineering, 2017, 039 (010): 1136-1140

[4] Huang Jianjun, Li Youmou, Liu Jing, et al. Design and implementation of automatic test system based on Python language [J]. Modern electronic technology, 2017 (04): 47-51

[5] Pu Tianhang. Research on instrument management and test system based on Python language $[\mathrm{J}]$. China instruments, 2020, no.347 (02): 42-45

[6] Zhou Xingye, Zhu Jianxin, Chen Xiang, et al. Study on dynamic insulation resistance detection method of battery pack for hybrid electric vehicle $[\mathrm{J}]$. Modern electronic technology, 2017, 40 (10): 121-124

[7] Zhang Xiangwen, Gao Guan. Real time online monitoring system for insulation resistance of electric vehicle power battery $[\mathrm{J}]$. Journal of Jilin University (Engineering Edition), 2017 (05): 1395-1402

[8] Ni Hongjun, Chen Xiang, Zhou Xingye, et al. Detection method of dynamic insulation resistance of vehicle battery pack: 2016

[9] Mao Xingyu. Detection of short circuit or grounding short contact of phase line in fully enclosed bus duct by DC balanced bridge principle [J]. Installation, 1998, 000 (005): 31-32

[10] [2015.249. Electric current detection method of electric bridge based on Zhang Xingyang University [2015]

[11] Shi Wei, Jiang Jiuchun, Li SuoYu, et al. Study on SOC estimation method of lithium iron phosphate battery [J]. Journal of electronic measurement and instrumentation, 2010, 24 (008): 769-774

[12] Dong Haiyang, Yang Yuxin, Luo Yu, et al. Design of insulation resistance detection system for electric vehicles based on STM32 [J]. Electronic design engineering, 2019, v.27; no.417 (19): 186-189 + 194 
[13] Zhu Feng. Design and development of instrument system software based on arm [D]. 2016

[14] Zhang Yue, Tao Linwei. Multichannel data acquisition system based on FPGA and STM32 [J]. Journal of Northwest Polytechnic University, 2020, v.38; No.182 (02): 128-135

[15] Chang Jiamin, Gao Fengmei, Wang Chongyang. Design of CAN bus communication between hybrid electric vehicle BMS and charger [J]. Industrial control computer, 2010, 23 (005): 45-46
[16] Zhou CF, Zhao Nan, Li Xin. An automated test system based on Python script language [J]. Journal of Nankai University (NATURAL SCIENCE EDITION), 2014 (05):

67-72 